\title{
Les sciences humaines et sociales en campagne : entre expertise et prophétie
}

Les interventions des chercheurs dans la presse durant les élections européennes de Mai 2014 (Le Monde, Le Figaro, Libération)

Humanities and Social Sciences Scholars on the Campaign Trail: Between Expertise and Prophecy. Analysing French HSS Scholars' Press Appearances In the 2014 European Election Campaign

\section{Constantin Brissaud et Éric Brun}

\section{OpenEdition} Journals

Édition électronique

URL : https://journals.openedition.org/bssg/659

DOI : 10.4000/bssg.659

ISSN : 2490-9424

Éditeur

Presses universitaires de Vincennes

Référence électronique

Constantin Brissaud et Éric Brun, «Les sciences humaines et sociales en campagne : entre expertise et prophétie », Biens Symboliques / Symbolic Goods [En ligne], 8 | 2021, mis en ligne le 20 mai 2021 consulté le 23 juillet 2021. URL : http://journals.openedition.org/bssg/659 ; DOI : https://doi.org/ $10.4000 /$ bssg. 659

Ce document a été généré automatiquement le 23 juillet 2021.

Biens Symboliques / Symbolic Goods 


\section{Les sciences humaines et sociales en campagne : entre expertise et prophétie}

Les interventions des chercheurs dans la presse durant les élections européennes de Mai 2014 (Le Monde, Le Figaro, Libération)

Humanities and Social Sciences Scholars on the Campaign Trail: Between

Expertise and Prophecy. Analysing French HSS Scholars' Press Appearances In the 2014 European Election Campaign

Constantin Brissaud et Éric Brun 
Fig. 1.

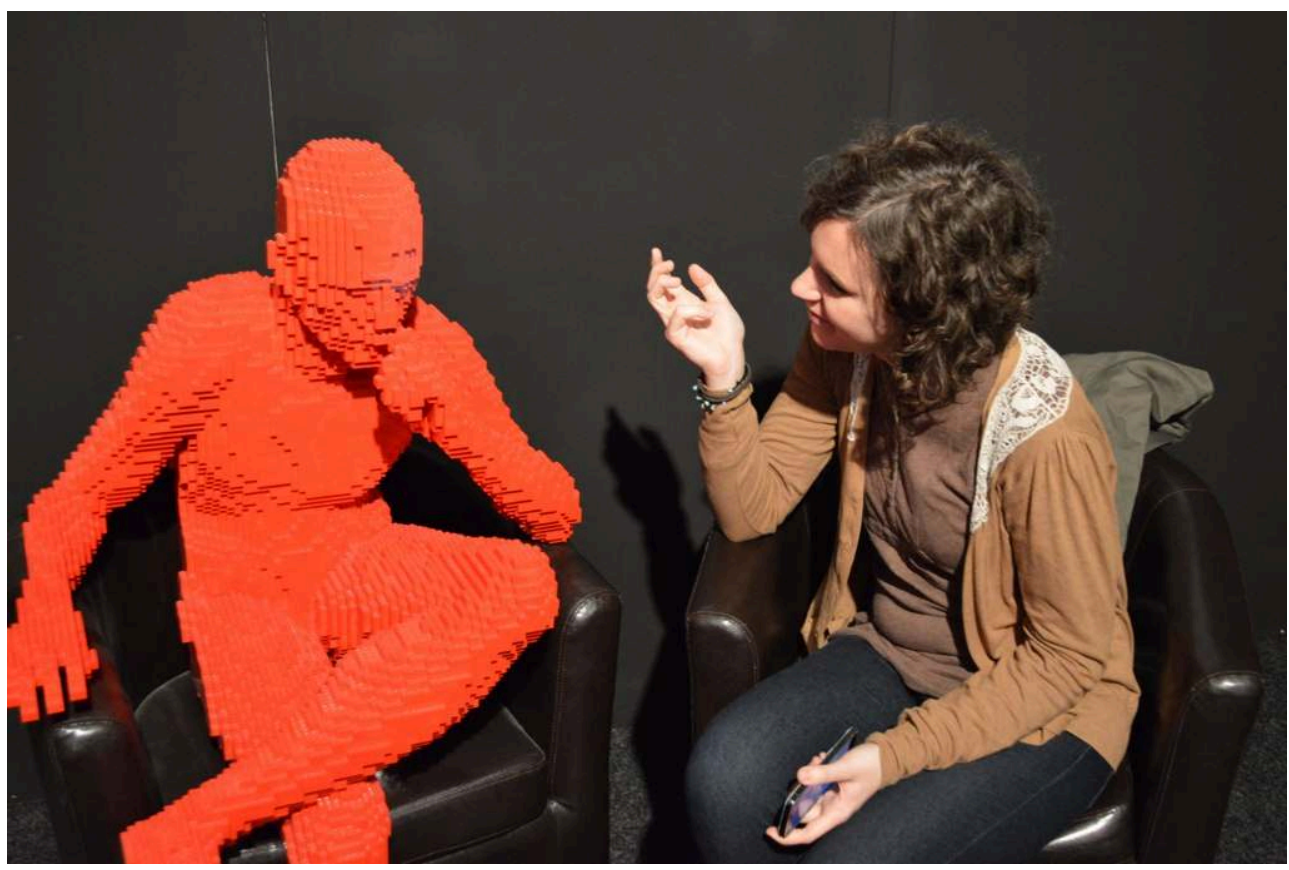

« Lego interview » par Matt Brown

Source : Flickr

\section{Introduction}

1 De même que l'emploi du terme « intellectuel $»^{1}$ a servi depuis l'Affaire Dreyfus en France à la constitution d'un groupe social très hétérogène (Charle 1990), les formes d'engagement des intellectuels se sont différenciées et opposées au cours de l'histoire (Matonti \& Sapiro 2009). À partir de la seconde moitié du XX $X^{\mathrm{e}}$ siècle, qui a vu notamment l'institution du format de la «tribune » (Jeanpierre 2016), la création de rubriques dévolues aux "points de vue » et "idées » dans la presse écrite, ainsi que le lancement d'émissions télévisées consacrées aux débats entre intellectuels (LensingHebben 2008) a profondément affecté leurs répertoires d'action disponible ainsi que les logiques de visibilité et de légitimation de leurs interventions.

Mais, si l'on pense généralement à la «victoire du champ journalistique qui s'est octroyé [...] une véritable fonction de juridiction sur le monde intellectuel » (François \& Neveu 1997 : 38 ; voir également Bourdieu 1984b) lorsqu'il est question des mutations contemporaines de la vie intellectuelle, une autre tendance majeure du $\mathrm{XX}^{\mathrm{e}}$ siècle mérite également d'être soulignée et interrogée : la spécialisation accrue du savoir, sur fond de lutte concurrentielle pour le monopole sur des territoires d'expertise (Abbott 1988). À partir de la fin du XIX ${ }^{\mathrm{e}}$ siècle, à la faveur de l'installation de nouvelles élites républicaines et d'une demande étatique d'expertises savantes (Delmas 2006), la montée en puissance du paradigme scientifique et l'institutionnalisation de plusieurs disciplines (Sapiro, Brun, Fordant 2018) viennent concurrencer la philosophie et les « humanités » (Soulié 2013 ; Joly 2017). On peut penser que ces évolutions favorisent une forme d'engagement politique particulière, celle de l'« expert », qui intervient dans l'espace public au titre d'une compétence spécialisée et reconnue par les institutions ${ }^{2}$, par opposition à l'engagement de l'intellectuel «universaliste » ou «total », 
intervenant à titre individuel au nom de valeurs générales (liberté, vérité, justice, beauté...) et potentiellement sur toutes les questions (politiques, économiques, sociales...) susceptibles d'impliquer de telles valeurs.

3 Souhaitant interroger cette distinction mais aussi celle effectuée par Max Weber - et reprise, à la suite de Bourdieu (Bourdieu 1971) qui l'a importé dans sa théorie des champs, par Gisèle Sapiro dans sa typologie des formes d'engagement des intellectuels au XXe siècle (Sapiro 2009) - entre l'idéal-type du "prêtre », dont l'autorité ne vaut que par la relation qui l'unit à une institution, et celui du "prophète ", qui tire son autorité d'un charisme attaché à sa personne, cet article cible une catégorie particulière d'intellectuels, les universitaires et chercheurs en sciences humaines et sociales (SHS) ${ }^{3}$. Accrédités par des institutions qui leur confèrent leur autorité savante, recevant pour la plupart d'entre eux un salaire de l'État, les universitaires et chercheurs que nous étudions pourraient a priori être rattachés à la figure du "prêtre" et à sa forme sécularisée dans le champ intellectuel, celle de l'expert, «qui établit son diagnostic suivant des procédures déterminées, quand la reconnaissance fondée sur le charisme prédispose [à l'inverse] au prophétisme » (Sapiro 2009: 11). La division académique sur la base de "disciplines» spécialisées du savoir, dotées d'un corpus de concepts, de méthodes, d'objets d'études propres (Gingras 1991 ; Heilbron 2004 ; Boutier et al. 2006), les situe là aussi - à l'exception de certains d'entre eux, comme les philosophes notamment - du côté des experts "spécialisés" par opposition aux intellectuels " universalistes".

4 Leurs modalités d'engagement politique sont-elles conformes à ce qu'on pourrait attendre d'eux au regard de ces quelques caractéristiques? Si l'on associe généralement la notion d'« expertise " à la réponse à une commande émanant d'une autorité, le plus souvent politique ou économique (Memmi 1989), l'éventail des modalités d'intervention politique des universitaires et chercheurs en SHS est évidemment plus étendu. En outre, ceux-ci se différencient sur certains points importants de l'idéal-type du prêtre : leur histoire, cristallisée dans leur statut (celui notamment d'enseignant-chercheur défini en 1984), leur autorise une forme d'indépendance ; en principe libérés - en tant que fonctionnaires - des fluctuations de la demande économique, ils ne sont pas non plus soumis au « devoir de réserve ${ }^{4}$ ». Leur répertoire d'interventions politiques disponibles comprend ainsi la possibilité de s'exprimer publiquement dans la presse, y compris sur des questions politiques et/ou en circonstance électorale. C'est précisément sur ce type d'interventions que se déploie notre analyse.

5 Comment caractériser les interventions dans la presse des savants en SHS ? La catégorie d'« expertise » permet-elle de décrire ce type d'engagements politiques et de saisir ce qui s'y joue? Plusieurs travaux portant sur les tribunes de presse ont montré non seulement que leurs auteurs se recrutaient en grande partie parmi les chercheurs et universitaires de différentes disciplines des SHS (Desintox 2004; Pinto 2002; Jeanpierre \& Mosbah-Natanson 2008), mais aussi que l'usage de ce format s'apparente le plus souvent à l'engagement de "l'intellectuel universaliste ». Notre article entend réactualiser ces analyses à partir d'un nouveau cas, celui des interventions de chercheurs ou universitaires en SHS dans trois des principaux titres de la presse quotidienne généraliste lors des élections européennes qui ont eu lieu le dimanche 25 mai 2014 en France et du 22 au 25 mai dans l'ensemble des pays de l'Union européenne (UE). Dans ce but, nous opérerons plusieurs déplacements de la focale d'observation, à 
commencer par la prise en compte, à côté des tribunes, d'un autre type d'articles de presse : les interviews (cf. encadré « la constitution du corpus »).

À quels titres ces interventions savantes durant les élections européennes ont-elles été effectuées? Comment se traduisent concrètement les revendications d'expertise savante dans la forme et le contenu de ces interventions? Peut-on observer, par exemple, une spécification des sujets abordés ou des manières de les aborder en fonction de la discipline d'appartenance des chercheurs considérés ? Et est-ce que cela suffit à en faire des discours « savants »? On montrera ici des formes de légitimation et de spécialisation qui font écho à l'idée d'« expertise savante ", mais qui n'empêchent au fond ni la sollicitation redondante des mêmes universitaires ou chercheurs les plus présents médiatiquement sur différents sujets, ni l'instrumentalisation des titres universitaires ou scientifiques par des auteurs situés à l'extérieur du champ académique. Corrélativement, nous relevons également l'adoption de postures plus classiques d'intellectuel "universaliste", et de recours à des registres d'expression relevant plutôt de l'interpellation "prophétique » au nom de valeurs menacées que du discours savant.

La constitution du corpus

Nous avons recueilli et analysé les interventions de chercheurs et universitaires en SHS parues entre le $1^{\text {er }}$ et le 31 mai 2014 dans trois quotidiens nationaux français (Le Monde, Libération, Le Figaro) retenus pour leur nature généraliste et plus ou moins représentative du spectre politique (Libération étant généralement classé à gauche, Le Monde au centre et Le Figaro à droite). Ont été retenus systématiquement tous les articles traitant de près ou de loin de « l'actualité » des élections européennes (ce qui inclut des articles dont la parution était objectivement liée à cette actualité sans pour autant qu'ils évoquent explicitement soit l'Europe, soit les élections proprement dites). Pour constituer ce corpus d'articles, nous avons effectué des recherches par mots-clefs sur les sites web des trois journaux retenus, consulté les bases de données Europresse et Factiva et, pour Le Monde, effectué un dépouillement systématique des éditions papier du quotidien sur la période. Ce corpus d'articles a ensuite été soumis à une double analyse quantitative (par l'emploi du logiciel d'analyse textuelle Iramuteq) et qualitative (par « lecture attentive ») et plusieurs informations biographiques ont été recueillies à propos de leur(s) auteur(s).

Le choix des titres de presse ainsi que de la séquence temporelle était déterminé par une problématique étrangère au présent article, portant sur l'émergence d'un espace intellectuel européen ${ }^{5}$ (Duller et al. 2017). Notons toutefois que le choix de trois titres de presse à l'exclusion d'autres médias (autres journaux, émissions télévisées ou radiophoniques, blogs, réseaux sociaux...) aurait été problématique s'il s'était agi de reconstituer l'espace des prises de position autour du processus de construction européenne. Il n'en est rien ici puisqu'il s'agit d'analyser les conditions et contraintes de mise en œuvre d'un type particulier d'engagement politique à disposition des "savants ", à savoir l'intervention publique dans la presse d'information généraliste française. Il s'avère du même coup préférable que le choix des articles ait procédé de leur insertion dans une séquence d'actualité plutôt que du fait qu'ils traitent d'une controverse unique (comme le font Duval et al. 1998). En effet, l'homogénéité relative du corpus analysé du point de vue du 
type d'intervention publique dont il relève se double d'une certaine diversité, du point de vue des sujets abordés.

Reste que le présent article expose un cas d'étude qui, en tant que tel, a vocation à susciter de futures comparaisons, en particulier avec des interventions publiques de savants en SHS sur d'autres supports d'expression et dans d'autres circonstances. Surtout, les analyses présentées ici gagneraient à être poursuivies par un travail d'enquête spécifique sur les opérations de sélection et de mise en forme des interventions « savantes " par les journalistes. À défaut, nous nous efforcerons de mettre en évidence ici certains signes des attentes et/ou interventions journalistiques, qui se livrent par exemple dans l'écart entre deux versions d'un même article (sur papier et en ligne), ou dans la mise en avant d'un auteur au sein d'un collectif d'auteurs, etc.

Pour ce qui concerne la sélection des auteurs, nous avons opté généralement pour un principe d'inclusion. En effet, notre corpus comprend une série d'auteurs dont la catégorisation comme " chercheurs ou universitaires en SHS » pose objectivement problème : sont ainsi inclus des magistrats, consultants, journalistes..., exerçant par ailleurs une activité d'enseignant dans une université ou une grande école et/ou une activité de recherche dans des organismes du type think tanks. En refusant de les exclure a priori, l'objectif était de donner toute sa place dans notre analyse à la porosité des frontières entre différents champs et aux enjeux stratégiques de présentation de soi (Collovald 1988). Il convenait autrement dit de ne pas décréter a priori les frontières légitimes de la catégorie de " savants ", afin de se donner les moyens d'observer l'état des rapports de force réglant la prétention à l'admission dans cette catégorie.

Notre corpus comprend ainsi quatre-vingt-sept auteurs pour quatre-vingt-un articles. Trente-neuf sont parus dans les éditions papier et/ou électronique $\mathrm{du}$ Monde, vingt-sept dans Libération et quinze dans Le Figaro. Quarante-cinq articles sont parus dans les trois semaines qui précèdent le jour de l'élection en France (le dimanche 25 mai) et trente-sept dans la seule semaine qui suit les élections. La parution d'un grand nombre d'articles se concentre sur les quelques jours avant et après le week-end des élections en France. Pour plus de la moitié, ces articles relèvent de «tribunes d'opinion » (quarante-sept), signées individuellement ou, quoique plus rarement, collectivement. Une autre catégorie d'articles très présente est les entretiens avec des savants considérés ici comme auteurs de ces articles (vingt-cinq). On compte également cinq articles s'inscrivant dans le cadre d'une chronique régulière et quatre articles relevant plutôt d'analyses (de sondages pré- ou post-électoraux), qui se différencient du genre de la " tribune » en ce que, d'une part, ils ne sont pas publiés dans les pages qui leur sont généralement consacrées, d'autre part ils ne visent pas à l'expression d'une « opinion », mais plutôt à l'analyse distanciée et objective.

\section{1. « L'expertise » comme mode de légitimation}

7 Les signatures des articles de notre corpus indiquent tantôt une discipline du savoir («économiste», «politologue», «docteur en science politique», 
« constitutionnaliste », « historien des idées », etc.), tantôt une fonction («professeur », " maître de conférences", "directeur d'études", etc.), elle-même garantie par une institution dont le nom exprime une prétention savante ("Sciences po », «Université », "Institut de recherches... ", etc.), tantôt les deux.

Le titre ne vient parfois qu'appuyer un capital symbolique déjà contenu dans le nom propre de l'auteur. Fait révélateur des formes de légitimité qui commandent l'accès à la presse généraliste, seuls les noms des économistes Jean-Paul Fitoussi, James K. Galbraith et Joseph Stiglitz sont par exemple mentionnés dans le titre d'une tribune collective parue dans Le Monde, les noms des neuf autres signataires, moins connus du lectorat français, étant renvoyés dans une courte note en fin d'article.

Il n'en reste pas moins que, même pour les plus célèbres, la présence d'un titre institutionnel et/ou la mention d'une discipline du savoir semble s'imposer. En effet, même si on ne peut exclure entièrement la possibilité que des universitaires ou chercheurs interviennent publiquement dans cette presse sans recourir à ces titres (de sorte qu'on ne les aurait pas identifiés), tout laisse à penser que ce cas est très rare. Quand bien même certains savants voudraient s'exprimer publiquement « en tant que simples citoyens", ils ne peuvent si facilement renoncer aux titres qui leur donnent accès à l'expression publique (Siméant $2002: 35$ ).

Dans certains cas, c'est aussi une "spécialisation " sur tel ou tel sujet qui est mise en avant. Pour un peu plus de $40 \%$ des articles de notre corpus, la signature de l'article associe au nom du signataire un ou plusieurs de ses ouvrages, dont le titre contribue à certifier l'autorité sur le sujet traité. Dans certains cas, plus rares semble-t-il, la spécialité thématique peut aussi être mentionnée explicitement. Le sociologue Vincenzo Cicchelli est ainsi présenté comme ayant «travaillé sur la socialisation cosmopolite", Yves Bertoncini comme ayant "consacré de nombreux ouvrages aux questions européennes », Vincent Tournier comme étant un "spécialiste des questions liées à la jeunesse et à la politique », etc.

11 Ces pratiques de mise en valeur de l'auteur par la mention d'un ouvrage et/ou d'une spécialité thématique ne semblent pas distribuées tout à fait au hasard. En particulier, elles apparaissent sensiblement plus fréquentes dans le cas des entretiens. Tout se passe donc comme si les journalistes, lorsqu'ils doivent répondre de leur principe de sélection d'un chercheur ou universitaire interrogé, avaient une propension à mettre en scène l'excellence de son expertise. Cette manière de légitimer les interventions dans la presse se double d'une association fréquente entre certains territoires de l'expertise et certaines disciplines du savoir.

\section{Un ancrage disciplinaire des interventions dans la presse}

Nous observons, dans le corpus d'articles sélectionnés, une fragmentation des sujets, de sorte que, en lieu et place d'une controverse commune autour des questions européennes, on a bien plutôt affaire à une série de débats faiblement articulés entre eux. Ces différents sujets s'organisent toutefois autour de deux séquences temporelles successives. Dans un premier temps (avant le 25 mai, jour des élections), il est principalement question de la réforme des institutions européennes, de la monnaie unique, de politique économique, de l'identité européenne, et, sans que ce thème soit 
pour autant prédominant, des "populismes" ou des "extrêmes» en Europe. En revanche, après l'annonce des résultats électoraux, l'essentiel des articles parus propose une analyse de la progression des scores électoraux du Front national (FN) ${ }^{6}$ en France, de ses causes et implications, de ce qu'il convient de faire pour enrayer cette montée en puissance, et/ou une analyse de la vie politique française (que va devenir le Parti socialiste (PS) ${ }^{7}$ ? comment va évoluer l'Union pour un mouvement populaire $(\mathrm{UMP})^{8}$ ? etc.).

13 La constitution, via l'emploi du logiciel Iramuteq (voir encadré «les fonctionnalités d'Iramuteq "), de classes de vocabulaires, met en évidence cet ordonnancement en fonction de différents angles ou sujets (figure 1). Les deux premières classes correspondent ainsi aux termes du débat post-électoral : la classe 2 ( $24 \%$ du total des mots utilisés) regroupe principalement le vocabulaire du champ politique français (dans sa définition partisane et électorale) et la classe 1 (18\%) fait référence plus largement à des "problèmes sociaux ». Les classes 4 et 3 renvoient quant à elles aux débats pré-électoraux sur les politiques économiques d'une part (classe $4: 16 \%$ ) et sur la construction européenne (élargissement de l'Union européenne, question ukrainienne, géopolitique, etc.) d'autre part (classe 3:24\% des mots employés). La classe 5 enfin, qui regroupe $18 \%$ de l'ensemble des mots employés dans le corpus, renvoie au lexique des institutions européennes - comme en témoignent les termes "Conseil ", " exécutif», ou "Commission", ainsi que les noms des deux candidats en lice pour la présidence de cette dernière institution, Jean-Claude Juncker et Martin Schulz.

Fig. 2.

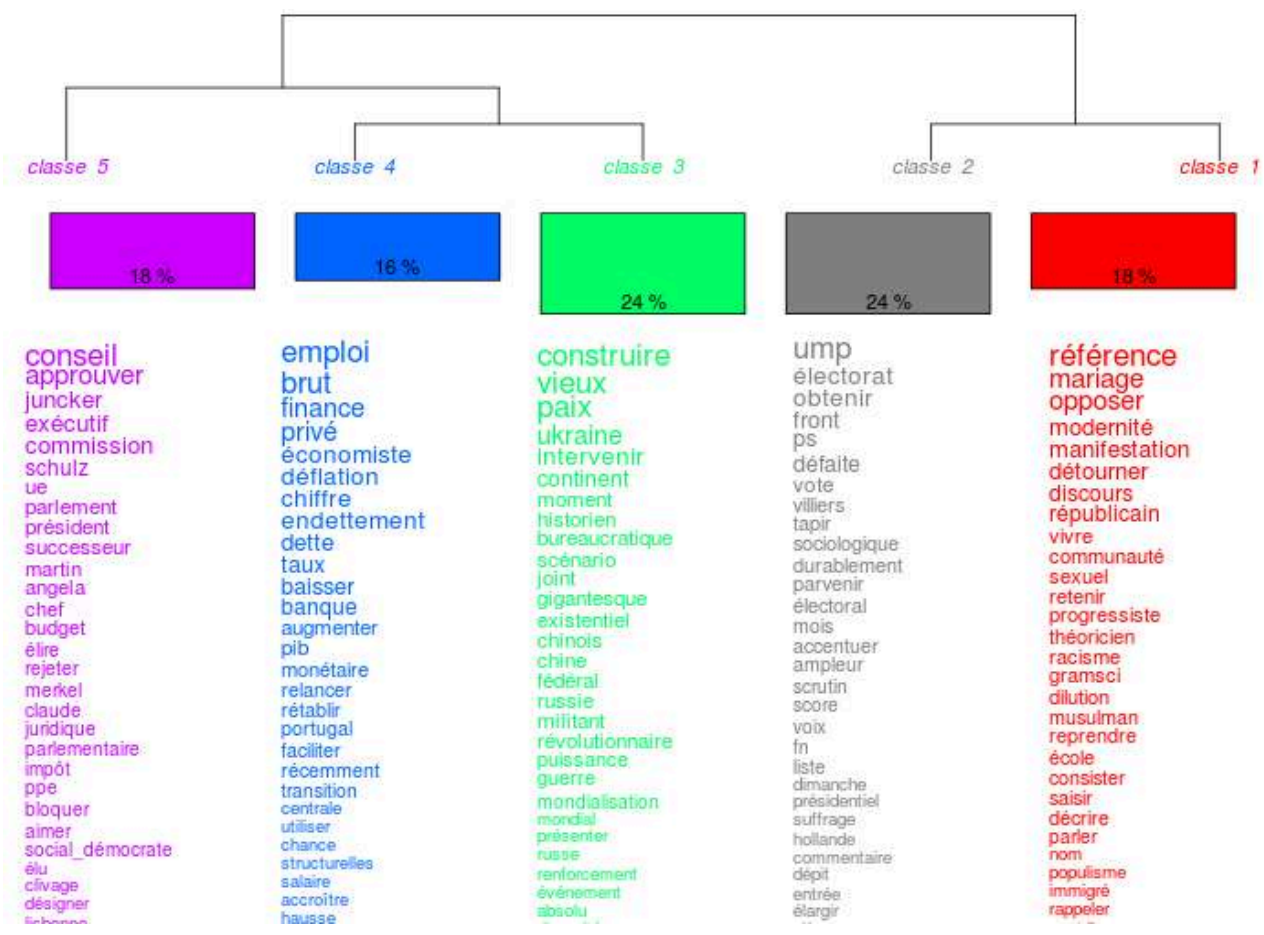

Classification hiérarchique descendante (CHD) des termes utilisés dans les articles

Source : Constantin Brissaud et Éric Brun

Or, le sujet abordé dans l'article tend lui-même à varier selon la discipline de l'auteur. Les économistes par exemple traitent avant tout de questions relatives à la politique 
économique et monétaire : il est question dans leurs articles des effets (jugés tantôt bénéfiques, tantôt néfastes, tantôt insuffisants) à attendre d'une sortie de l'euro. Il y est question également de la pertinence des politiques économiques (en termes de crise économique, de croissance, d'emploi, etc.), budgétaires, monétaires, mises en place par la Commission européenne ou la Banque centrale, ou impliquées par les traités qui régissent l'Union européenne.

Quant aux thèmes relatifs à la construction européenne, ou au fonctionnement des institutions européennes, ils mobilisent une partie importante des quelques étrangers de notre population - tels que le politologue Ivan Krastev (interrogé par Le Monde sur l'élargissement de l'Union européenne), le linguiste Raffaele Simone (interrogé par Libération sur « la crise actuelle de l'Europe »), ou encore le théoricien de la littérature Tzvetan Todorov (publiant une tribune dans Le Monde qui appelle à « révolutionner les institutions européennes ») -, ainsi que la plupart des politologues, ou des spécialistes des «questions européennes» et, finalement, la majeure partie des enseignants de Sciences Po Paris présents dans notre population.

Les politologues tendent par ailleurs à monopoliser deux types d'articles dans notre corpus: le premier consiste à établir, au lendemain de l'annonce des résultats électoraux, les nouvelles coordonnées de la vie politique française (que va devenir l'UMP ? quel sera le candidat du PS en 2017 ? etc.) telles que dévoilées par ces élections ; le second type d'articles consiste à interpréter, chiffres à l'appui, les sondages ou résultats électoraux (articles qui sont alors publiés dans les pages principales et non dans celles consacrées aux « débats » et " points de vue »). À côté de Martial Foucault et Pascal Perrineau (tous deux membres du Cevipof, le Centre de recherches politiques de Sciences Po, qui s'est fait une spécialité de l'analyse électorale), c'est un autre chercheur en science politique, Jean-Yves Dormagen qui, en tant que fondateur de l'observatoire du changement politique ${ }^{9}$, signe un article de ce type dans Libération suite à l'annonce des résultats.

Quant aux sociologues, ceux qui interviennent avant les résultats des élections le font surtout pour traiter de "l'identité européenne ", thème qu'ils partagent alors avec des philosophes. Ils sont également très présents dans les quelques jours qui suivent les élections pour des articles qui traitent de l'extrême-droite et des causes de sa progression. C'est par exemple le cas de Luc Boltanski et Arnaud Esquerre, Philippe Corcuff, Raphaël Liogier, Michel Maffesoli ou encore Michel Wieviorka, auxquels on pourrait ajouter des politologues privilégiant une approche plus sociologique du fait politique comme Sylvain Crépon et Alexandre Dézé. Là encore, les sociologues partagent ce thème avec des représentants d'autres disciplines: des géographes, quelques économistes et philosophes. Dans le même temps, plusieurs spécialistes des médias comme François Jost et Philippe Riutort traitent plus spécifiquement de la contribution des médias aux scores du FN.

Cet ordonnancement des sujets privilégiés par les différentes disciplines de SHS oppose en résumé trois pôles, celui des économistes, celui des politologues, et un dernier pôle plus indifférencié regroupant des représentants de différentes sciences humaines (sociologie, philosophie, histoire, géographie...). Il se repère également dans des variations disciplinaires des lexiques employés. Ainsi, sur deux plans factoriels réalisés à partir des termes employés plus ou moins fréquemment par les auteurs, on remarque à nouveau des oppositions disciplinaires nettes. 
Les fonctionnalités d'Iramuteq

Les figures 2, 3 et 4 ont été obtenues grâce au logiciel d'analyse textuelle Iramuteq, développé par Pierre Ratinaud au Laboratoire d'études et de recherches appliquées en sciences sociales de l'Université de Toulouse 3-Paul Sabatier (Iramuteq est l'acronyme de « Interface de R pour les analyses multidimensionnelles de textes et de questionnaires »). Tous les textes des articles ont été saisis dans le logiciel, en y associant des balises (i.e. le nom de l'auteur, la date et le journal de parution de l'article). Iramuteq opère alors une analyse factorielle des correspondances sur un tableau de contingence croisant les mots présents dans les articles et les articles (ou les noms des auteurs des articles). Le logiciel projette ensuite sur un plan factoriel les noms des auteurs en fonction des mots qu'ils utilisent. Deux auteurs proches sur le plan factoriel partagent donc l'usage d'un grand nombre de mots. Par « mots ", il faut ici entendre « lemmes » : chaque mot a été réduit à sa racine pour être associé aux autres mots de la même famille. Par exemple « élire » (et ses conjugaisons), « élections ", « élection », « électeurs » et « électeur » sont regroupés dans une même famille via la lemmatisation. En outre, les mots les plus courants de la langue française comme « de », " et ", « à », etc. sont omis de l'analyse et ne participent donc pas à la construction des axes (pour plus d'information sur la lemmatisation, voir Mayaffre 2005). Iramuteq permet en outre de procéder à une classification hiérarchique descendante (CHD) des termes utilisés. Ainsi, les mots le plus souvent présents ensemble dans un même texte vont constituer une classe de la CHD, que l'on peut analyser comme un thème du corpus. L'analyse par segments de textes caractéristiques donne, pour chaque classe constituée, les extraits d'articles les plus typiques de la classe, et donc les dates et auteurs les plus caractéristiques de cette classe.

On trouve à l'ouest de la figure 2 essentiellement le langage des économistes (« taux », « emploi », etc.), ce qui correspond, sur la figure 3, à la position de la majeure partie des économistes de notre population. De même, on trouve au nord-est de la figure 2 un lexique associé à la vie politique et plus encore électorale française («suffrage», «vote», etc.), avec ses échéances passées («2009») ou à venir («2017», " présidentiel»), ses partis ( UMP», «frontiste») et stratégies (" allié »); ce qui correspond là aussi sur la figure 3 à une forte concentration de politologues ${ }^{10}$; au sudest, sont davantage représentées d'autres sciences humaines (avec des sociologues, philosophes ou encore des psychanalystes comme Stéphane Habib), qui ont en commun l'emploi de termes ayant trait à la société (" génération », " jeunesse », " mariage ») ou à la communication (« information », « parole », « média »...). 
Fig. 3.

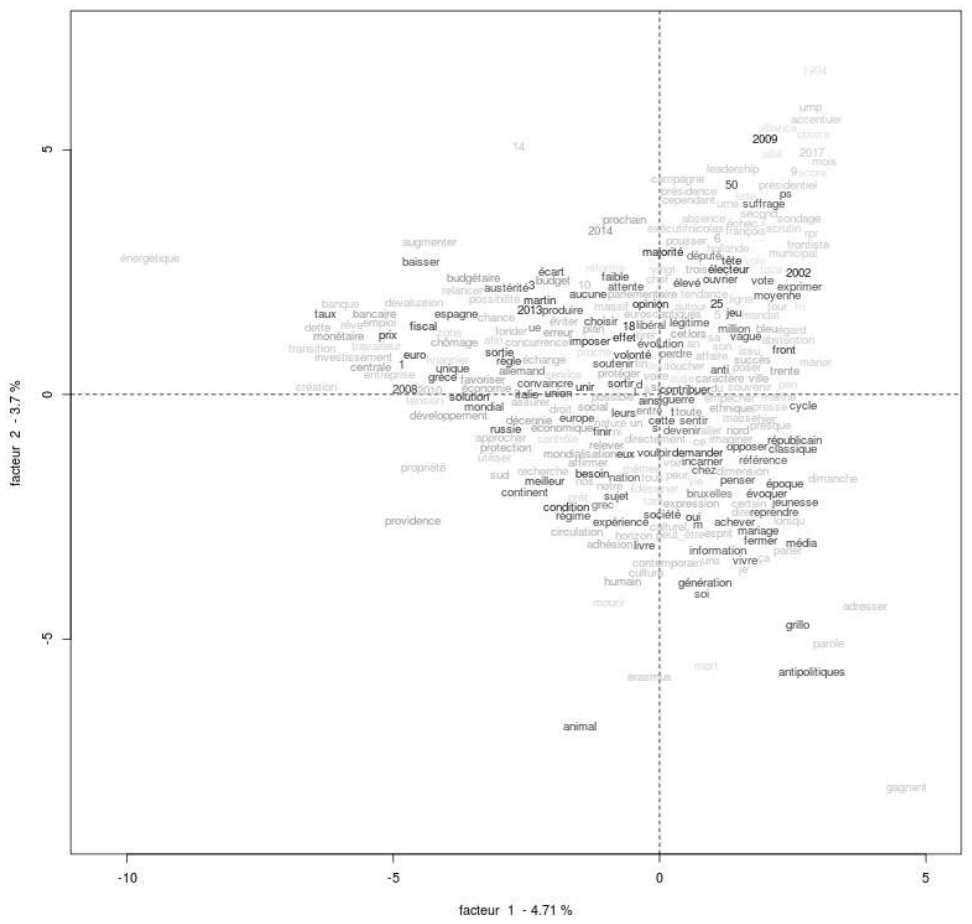

Plan factoriel (AFC) des termes employés dans les articles du corpus Source : Constantin Brissaud et Éric Brun 
Fig. 4.

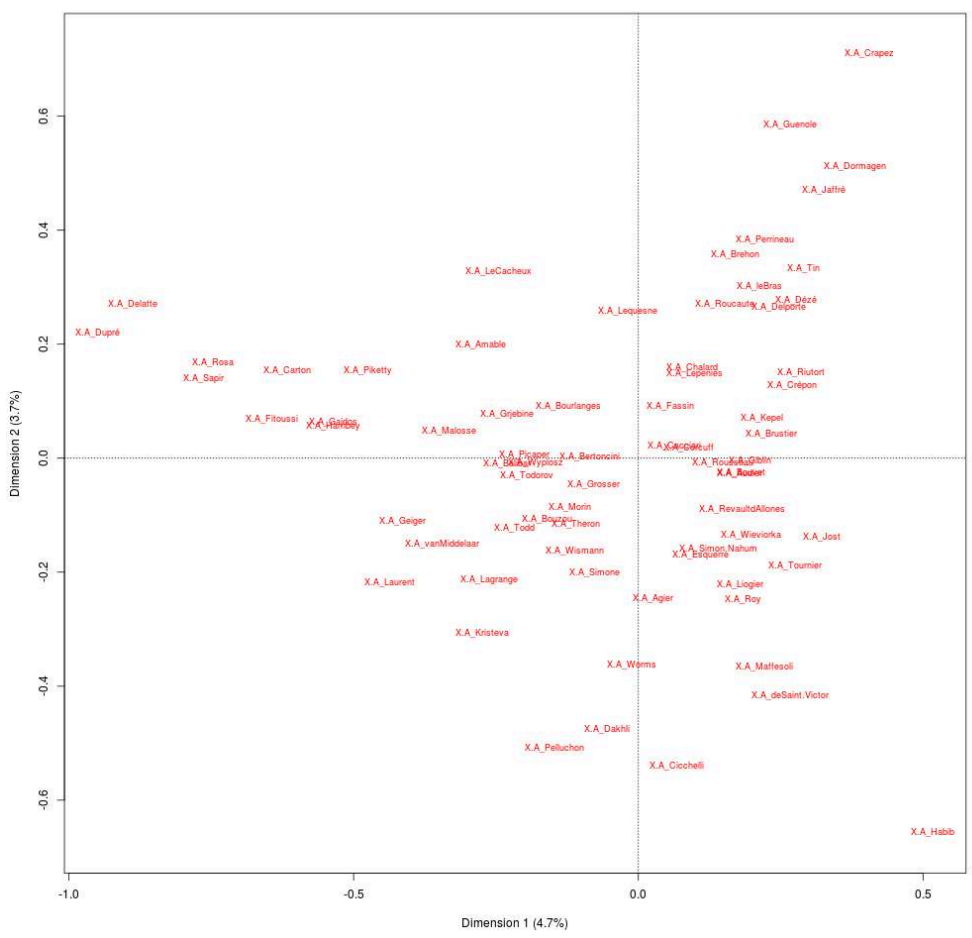

Plan factoriel (AFC) des auteurs en fonction des termes utilisés dans leurs articles Source : Constantin Brissaud et Éric Brun

Cette correspondance relative entre disciplines d'appartenance des auteurs et sujets/ lexiques des articles permet-elle de conclure à l'adoption actuelle, chez les universitaires et chercheurs en SHS, lorsqu'ils interviennent dans la presse généraliste, d'un modèle de « l'expert » spécialiste d'une discipline ou d'un sujet donné ? Il convient ici de faire la part des choses: le découpage disciplinaire des thèmes et des vocabulaires, s'il reflète une répartition des territoires d'expertise entre disciplines, n'interdit pour autant en rien l'emploi de postures plus classiques d'engagement dans les articles considérés. Pour s'en rendre compte, il convient d'interroger les registres argumentatifs employés.

\section{Entre préconisations et prophéties}

Quand bien même les articles qui composent notre corpus sont parus en contexte de campagne électorale, la politique y apparaît le plus souvent sous une forme feutrée : rares sont les interventions qui engagent explicitement leur auteur dans les enjeux les plus directement partisans ${ }^{11}$. À l'inverse, on a affaire plus souvent à des préconisations adressées aux responsables politiques et/ou à l'opinion publique, en faveur tantôt de réformes claires, tantôt de réorientations politiques plus générales: " démocratiser les institutions européennes ", "créer un parlement de la zone euro ", " changer le logiciel idéologique de la gauche », «faire marcher la planche à billets ", "renoncer au journalisme de sondage ", etc. Les auteurs recourent alors au registre directif ou incitatif à travers l'emploi de formules comme: «les États membres 
devraient avoir le courage... », « osons... », " pour une... », « nous demandons que... », " c'est uniquement en... que les futures initiatives pourront... », etc.

Si ce registre de la préconisation peut s'interpréter comme la conséquence du processus historique d'autonomisation d'un champ académique à l'égard du champ politique (Bourdieu 1988 ; Duclert 1999 ; Matonti 2002), on ne saurait en déduire que les interventions des chercheurs et universitaires dans la presse répondent ici aux codes de la production dite scientifique. Dans le cas des tribunes (qui font appel à une conception du débat public comme confrontation d'une pluralité d'opinions et non comme objectivation méthodique et contrôlée de faits) aussi bien que dans celui des entretiens avec un "expert", on a affaire à des genres dont la codification fait intervenir avant toute chose les contraintes propres au champ journalistique (concision, clarté, «actualité » du sujet, etc.). On le remarque au fait que ces articles s'avèrent peu propices au déploiement d'un appareil d'administration de la preuve ${ }^{12}$.

Rares sont par exemple les articles de notre corpus qui mobilisent des données chiffrées ou cartographiques (ce qui, il est vrai, n'est pas une garantie de scientificité), à l'exception des quelques articles qui portent sur la politique économique ou de ceux, déjà évoqués, qui ont pour objet un commentaire "savant» des sondages et des résultats électoraux. Quant aux illustrations, quand elles existent, elles sont le plus souvent des photographies (plus rarement des dessins) se contentant de symboliser le sujet traité : drapeau européen, sigle euro " $€$ », photos du Parlement européen, sans parler des photos de Marine Le Pen (présidente du Front national) omniprésentes après l'annonce du résultat.

24 Et quand bien même les interventions semblent souvent travaillées par l'habitus disciplinaire de l'auteur (au regard du vocabulaire adopté, des sujets abordés), les registres argumentatifs déployés se laissent en revanche difficilement saisir sous la forme de purs diagnostics " dépassionnés ». Les articles de notre corpus recourent en effet fréquemment à des formulations qui font davantage écho à la figure du "prophète» interpellant le public sur le sens d'une crise en cours, qu'à celle du «prêtre » établissant un diagnostic à partir de procédures stabilisées. C'est le cas surtout après l'annonce du résultat des élections, alors que les sociologues (ainsi que les philosophes) occupent une place importante dans les articles parus: à la faveur du sentiment de crise - c'est alors la première fois que le Front national arrive en tête d'une élection nationale (avec près de $25 \%$ des suffrages), devant l'UMP (21\%) et le Parti socialiste au pouvoir (14\%) - une tonalité prophétique s'impose dans la plupart des articles. Les scores obtenus par le FN y sont en effet analysés comme un péril imminent pour la démocratie française (l'échiquier politique est "ébranlé », la nation "plongée dans un état de dépression électorale ", on assiste à un "dépérissement de la démocratie", à "une fracture ", un "danger majeur", "il devient urgent de..." " avant qu'il ne soit trop tard", etc.). Dès lors, le discours des uns et des autres vise principalement à le conjurer : il s'agit de répondre à cette crise en lui donnant son sens (il est question de "signe des temps »), c'est-à-dire aussi sa direction : "l'heure n'est plus à...» dit l'un; "tôt ou tard, la société...» assure un autre; «le temps de la refondation idéologique de la gauche est sans doute venu» affirme un troisième. Notons que le "sans doute ", présent dans le corps du texte, disparaît dans le titre de cet article/entretien paru dans Le Figaro, signe que les attentes journalistiques contribuent certainement à renforcer cette dimension prophétique. 
Fig. 5.

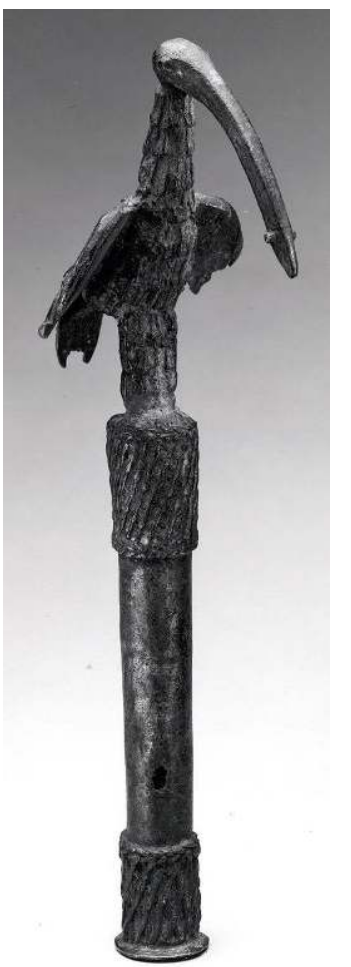

«Oiseau de Prophétie (ahianmwen-oro) » (instrument de musique, Nigeria, XVIe-XIXe siècles)

Source : New York, Metropolitan Museum

\section{S'exprimer dans la presse : une affaire d'hommes de pouvoir?}

De même que «la question de la définition de l'intellectuel ou, mieux, du travail proprement intellectuel, est inséparable de la question de la délimitation de la population qui peut être admise à participer à cette définition » (Bourdieu 1984a : 285), analyser les caractéristiques des interventions des savants en SHS dans ce secteur de la presse suppose de prendre en compte non seulement les contraintes de leur format, mais également les propriétés qui singularisent la population d'auteurs concernés.

À l'examen de la population d'auteurs des articles sélectionnés, on ne saurait nier une certaine diversité des profils, sensible par exemple à travers la présence d'individus, souvent plus jeunes que les autres, ne disposant que de peu de notoriété publique ou de reconnaissance extra-académique et dont le titre savant est, autrement dit, le seul à même de justifier leur intervention publique. Mais leur présence demeure assez faible ${ }^{13}$, et ne suffit guère à contredire le constat ancien d'une forte sélectivité sociale de l'accès à la presse nationale généraliste.

En effet, les universitaires et chercheurs qui composent notre population se recrutent en grande partie parmi certaines fractions spécifiques du champ académique : à la faveur d'une carrière généralement bien avancée (leur moyenne d'âge s'établit à 57 ans au moment de l'étude et les plus de 60 ans regroupent à eux seuls près de la moitié des auteurs) et de leur appartenance au sexe masculin (pour 91 \% d'entre eux), ils cumulent 
souvent plusieurs titres, occupant ainsi une position sécante entre champ intellectuel et champ du pouvoir (Bourdieu 2011: 126; Denord et al. 2011: 24). Ainsi, sur les soixante-dix-sept individus français ou travaillant en France, qui composent euxmêmes la très grande majorité des auteurs d'articles recensés dans notre corpus ( $C$. encadré "Un espace de débat européen?»), vingt-six figurent dans le Who's who in France (soit $34 \%$ ), vingt-deux ont reçu la légion d'honneur (soit près de $30 \%$ ) et plusieurs participent en parallèle à divers organismes chargés de l'expertise d'État: commissions ministérielles, Conseil d'analyse économique (CAE), Haut-Conseil à l'intégration, Haut-Conseil de la famille, etc. De plus, la moitié environ exerce ses activités de recherche dans les institutions françaises perçues comme les plus prestigieuses dans le domaine des sciences humaines et sociales. À l'inverse, seuls $14 \%$ sont rattachés à une université non-parisienne (figure 5).

Fig. 6.

\begin{tabular}{|c|c|c|}
\hline $\begin{array}{c}\text { Rattachement académique } \\
\text { principal }\end{array}$ & Effectifs & $\%$ \\
\hline CNRS, EHESS, ENS & 21 & 27 \\
\hline IEP & 18 & 25 \\
\hline Université parisienne & 19 & 14 \\
\hline Université de province & 11 & 11 \\
\hline Autre & 8 & 100 \\
\hline Total & 77 & \\
\hline
\end{tabular}

Effectifs (et pourcentage) des auteurs français par rattachement académique

Source : Constantin Brissaud et Éric Brun

Un espace de débat européen?

Une dizaine d'individus de notre population n'ont pas la nationalité française (14\%) auxquels on pourrait ajouter quelques Français travaillant à l'étranger. Cette internationalisation demeure somme toute assez faible dans le cadre d'un corpus constitué le mois des élections européennes. Remarquons à ce propos que les étrangers présents de notre corpus ont le plus souvent été « sollicités » sous la forme d'interviews (il ne s'agit donc pas de tribunes spontanément proposées) ou de traductions d'articles parus dans des journaux étrangers. De plus, le recentrement sur l'espace national des débats apparaît d'autant plus fort après l'annonce des résultats des élections : seuls quelques articles adoptent alors un angle plus international, comme celui de Charles Wyplosz (professeur d'économie 
internationale à l'Institut des hautes études internationales et du développement à Genève).

Surtout, la présence de quelques intellectuels étrangers dans la presse quotidienne nationale apparaît soumise elle-même à de fortes distorsions : loin d'être de parfaits inconnus en France au moment des élections européennes, plusieurs d'entre eux ont déjà eu un ouvrage paru en français chez un grand éditeur : chez Flammarion en 2006 pour Ian Buruma, chez Albin Michel en 2012 pour Heinz Wismann (qui est par ailleurs directeur d'études à l'EHESS), chez Gallimard pour Luuk Van Midelaar, chez Gallimard et au Seuil pour Wolf Lepenies, etc. De même, nombre d'entre eux (Krastev, Buruma, Müller, de Schütter, Galbraith, Stiglitz) ont également en commun d'avoir collaboré à Project Syndicate ${ }^{14}$. Cette importance des intermédiaires (éditeurs, organes et chroniqueurs de presse) susceptible d'expliquer la présence de ces auteurs dans la presse française laisse penser que cette ouverture relative de la presse nationale à quelques intellectuels étrangers ne saurait être tenue pour une preuve en soi de l'existence d'un espace intellectuel européen, mais gagnerait à être resituée dans le cadre de stratégies éditoriales et journalistiques à l'échelle d'espaces nationaux.

Notons également que toutes les disciplines des SHS ne sont pas également représentées dans notre population des auteurs d'articles. Ainsi, si l'on se borne à comptabiliser la discipline mentionnée dans la signature ou dans la présentation de l'auteur de l'article - mode de calcul qui conduit d'une part à exclure une trentaine d'auteurs pour lesquels aucune discipline n'est mentionnée, et à comptabiliser à l'inverse plusieurs fois des individus lorsqu'ils sont rattachés à plusieurs disciplines l'économie arrive en tête (13), précédant la philosophie (12), la science politique et la sociologie (11 chacune), puis l'histoire (7), loin devant la géographie (2), la démographie (2), la psychanalyse (2), ou encore l'anthropologie (2).

Certes, nous avons vu que certains sujets tendent à «mobiliser » en priorité certaines disciplines, de sorte que le fait que la séquence temporelle considérée soit celle d'élections affecte nécessairement le rang occupé par les différentes disciplines du savoir dans notre population d'auteurs. On ne saurait pourtant expliquer par là le décalage important entre les disciplines très représentées et celles qui le sont très peu, d'autant que les disciplines qui se dégagent ici sont aussi celles qui se dégagent d'autres travaux portant sur les tribunes de presse et dont le corpus n'était pas limité par cette circonstance des élections européennes (Jeanpierre \& Mosbah-Natanson 2008). On peut donc y voir également le signe d'une inégale répartition, selon la discipline d'appartenance, de la capacité à être considéré comme " expert " par les journalistes.

Enfin, par-delà cette diversité (toute relative) des appartenances disciplinaires, notre population se caractérise par la forte présence en son sein (à hauteur de $36 \%$ ) d'anciens élèves des Instituts d'études politiques (IEP), et notamment de l'IEP de Paris, dit "Sciences Po » [les IEP sont des établissements prestigieux d'enseignement supérieur, accessibles sur concours]. Cette propriété concerne certes avant tout les politologues, mais elle réunit également, entre autres, de nombreux économistes (André Grjebine, Gérard Lafay, Jacques Sapir...), plusieurs sociologues, ou encore un historien et un démographe. On peut penser que, redoublant en quelque sorte l'effet de la socialisation masculine et de l'avancée en âge (social), le passage par cette institution de formation des élites politiques, intellectuelles et administratives, dont on connaît l'importance 
qu'elle accorde à la " culture générale » (Bourdieu 1989 ; Garrigou 2001), est propice à l'intériorisation d'une disposition à se sentir habilité à intervenir publiquement sur des questions politiques selon des perspectives accordées avec les manières de voir le monde (et le rôle de l'intellectuel) parmi l'élite journalistique qui est bien souvent elle aussi passée par cette institution (Lafarge \& Marchetti 2011).

\section{Des intellectuels tournés vers le débat public}

De manière cohérente avec ce constat, les universitaires et chercheurs qui composent notre population ont souvent en commun d'incarner une conception particulière de l'activité savante, fortement orientée vers les débats sur la vie politique ou l'actualité. À l'examen de leurs pratiques de publication antérieures, on constate ainsi que plus de la moitié d'entre eux ont publié au cours de la période 2000-2014 au moins un article dans un groupe restreint de revues intellectuelles "généralistes" comprenant Esprit, Le Débat, Commentaire, Les Temps modernes, Mouvements, Vacarme, Multitudes, Revue projet et Cités. Même à ne prendre en compte que l'une d'entre elles, Le Débat, lancée en 1980 par Pierre Nora (et stoppée en 2020) pour, selon ses propres mots, « lutter [...] contre la réduction médiatique d'un côté, la spécialisation universitaire de l'autre ${ }^{15}$ ", près de vingt individus de notre population ont publié dans ses pages entre 2000 et 2014.

On remarque en outre que les individus de notre corpus ne sont qu'exceptionnellement des novices en matière d'intervention dans les médias, y compris dans les médias audiovisuels. Illustration du pouvoir prescripteur que confère la présence dans les médias télévisés (Bourdieu 1996), parmi les soixante-dis-sept auteurs français ou travaillant en France, soixante-quatre sont intervenus au moins une fois à la télévision française entre 2000 et 2014 (soit plus de $80 \%$ ). On retrouve du reste ici un phénomène aujourd'hui bien décrit de sollicitations redondantes et de surexposition médiatique d'un petit groupe d'individus (Jeanpierre \& Mosbah-Natanson 2008; Malherbe \& Luce 2017). Ce phénomène se signale d'emblée par le fait que, parmi les individus qui constituent notre population, sept sont présents pour plusieurs articles à la fois (et ce alors même que la période retenue dans notre étude de cas ne dure qu'un mois), comme le consultant Yves Bertoncini ou encore le politologue Thomas Guénolé. Le même phénomène se signale, d'ailleurs, par un "effet Mathieu " (Merton 1968) lorsqu'on observe le nombre de renvois obtenus pour chaque auteur de notre population sur la période 2000-2014 en interrogeant le catalogue de l'INA, afin de calculer l'ensemble de leurs interventions dans les médias audiovisuels et radiophoniques: plus on se rapproche du petit groupe de ceux qui sont intervenus le plus, plus le nombre d'interventions comptabilisées augmente rapidement (figure 6). 
Fig. 7.

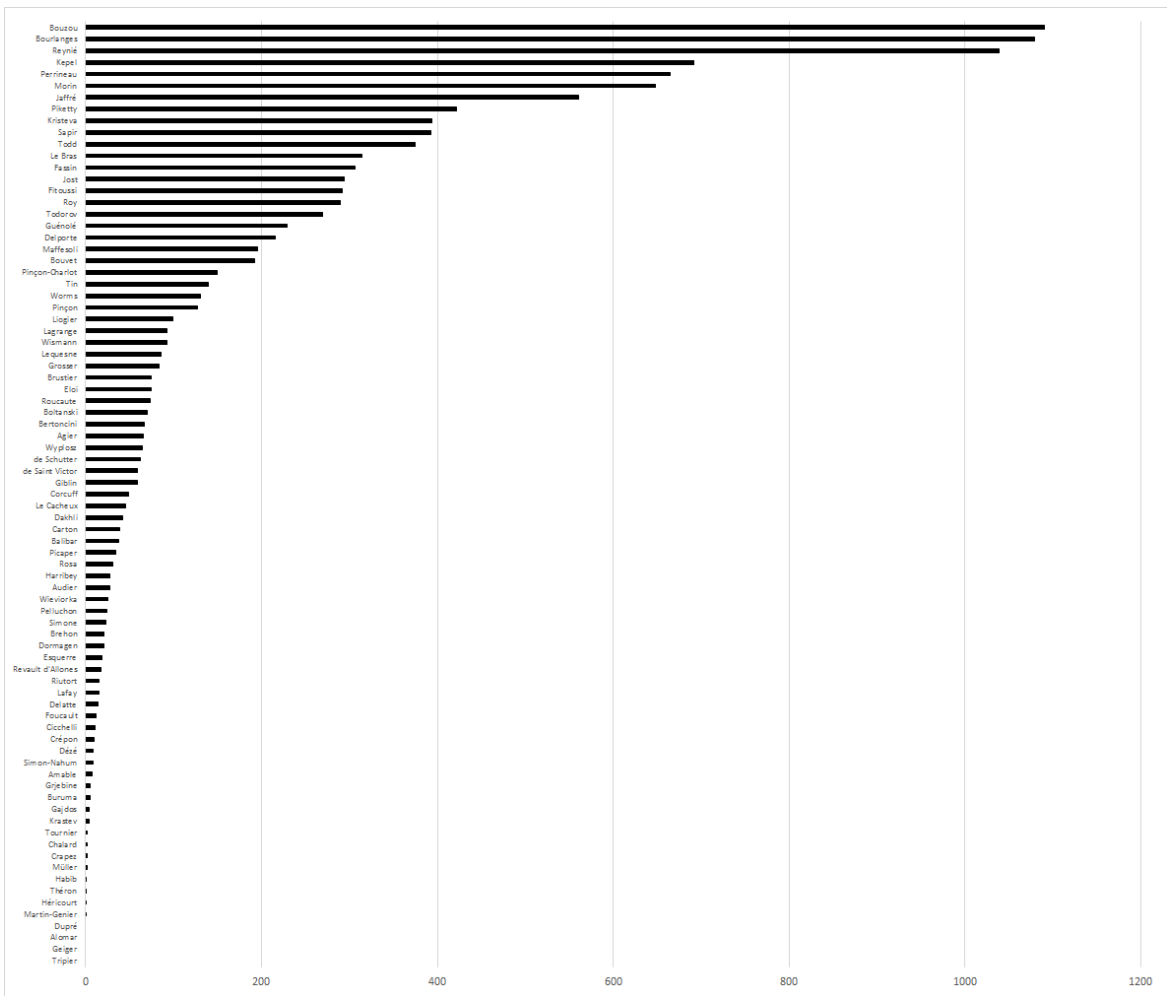

Nombre d'interventions réalisées à la télévision et radio françaises de 2000 à 2014 pour chaque auteur de notre population

Source : Catalogue de l'Institut national de l'audiovisuel

Le petit groupe des auteurs les plus médiatisés dans notre population comprend pour l'essentiel des politologues (ou des économistes) comme Nicolas Bouzou (1 090 entrées sur la période), Jean-Louis Bourlanges (1 079), Dominique Reynié (1039) - ce qui renvoie souvent chez eux à une activité de chroniqueur régulier dans une émission télévisée ou à la radio ${ }^{16}$. D'autres auteurs parmi notre population se caractérisent de leur côté plutôt par un nombre élevé d'interventions dans un genre particulier d'émissions, les «talk-shows » (Malherbe \& Luce 2017). Ainsi, sur la période 2010-2015, le sociologue Michel Maffesoli a été invité 22 fois, le politologue Gilles Kepel 23, le démographe et historien Emmanuel Todd 25, l'économiste Thomas Piketty 28, le politologue Thomas Guénolé 30 et le sociologue Éric Fassin 96.

Enfin, lorsqu'ils interviennent dans ce secteur de la presse que sont les principaux quotidiens nationaux, les universitaires ou chercheurs de notre population correspondent parfois assez peu à la figure de « l'expert » ou de l'intellectuel spécifique selon la notion de Foucault. Si nous n'avons pas reconstitué l'ensemble des parcours d'intervention publique des auteurs de notre population, notre corpus lui-même fournit plusieurs exemples de prises de positions hors de ce qu'on peut considérer comme leur domaine d'expertise. On pense ainsi par exemple au démographe et historien Emmanuel Todd assurant en entretien que "l'euro est un échec avéré ", ou encore à l'économiste Nicolas Bouzou expliquant le score élevé obtenu par le Front national par "l'obsession antimondialiste » des "perdants de la mondialisation ». La notion d'" expert total » récemment introduite pour parler des économistes, dont la boîte à outils conceptuels permet d'avancer un discours d'apparence savante sur un 
nombre très varié de sujets (Lebaron 2017), apparaît ainsi plus appropriée pour caractériser plusieurs des auteurs de notre population.

Fig. 8.

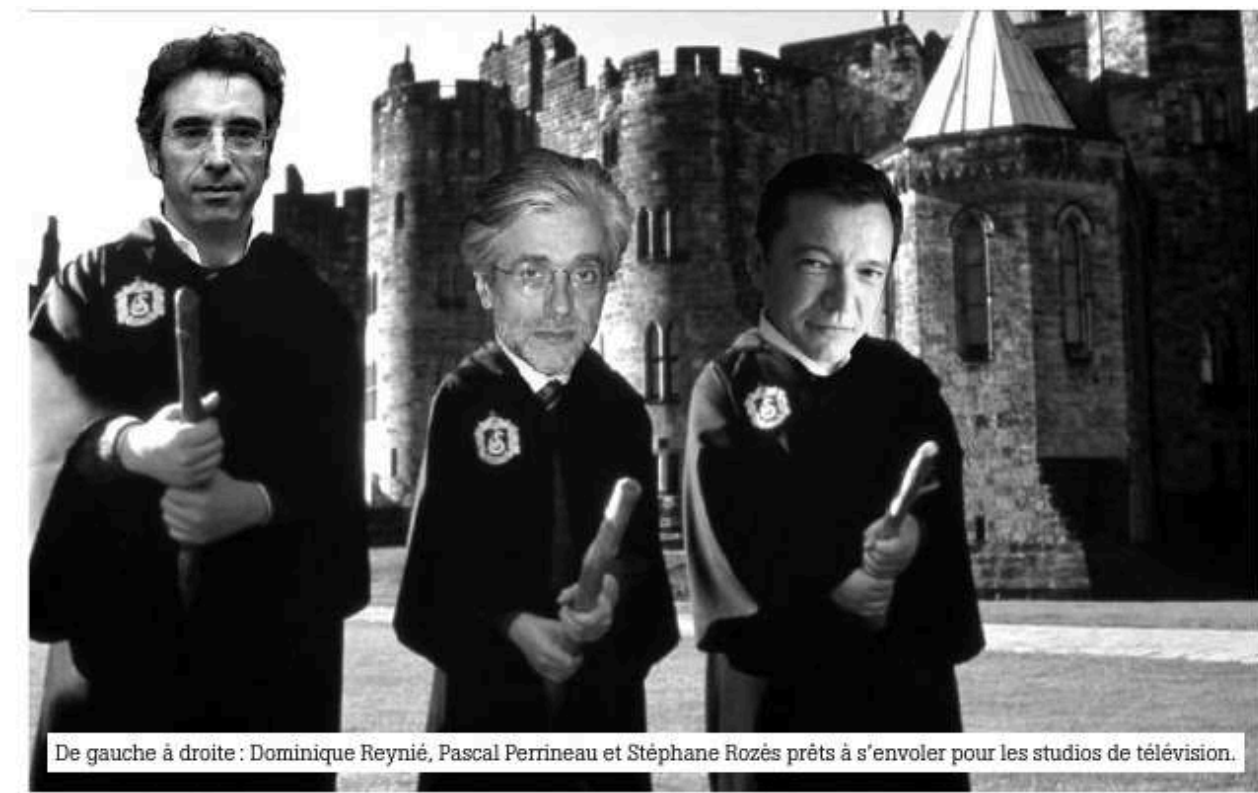

«Dominique Reynié, Pascal Perrineau et Stéphane Rozès prêts à s'envoler pour les studios de télévision»

Source : Le Plan B, n²3 (mai-juin 2010), journal de critique des médias (2006-2010)

\section{6. À l'intersection des champs académique, politique et journalistique}

Cet aperçu des quelques caractéristiques saillantes de notre population de chercheurs et universitaires risquerait d'être incomplet si l'on omettait de mentionner qu'on a souvent affaire ici à des individus combinant plusieurs appartenances institutionnelles voire professionnelles : à la fois universitaire et chroniqueur, journaliste, essayiste, magistrat, dirigeant d'entreprise, consultant, conseiller politique... Pour le dire autrement, la convocation, dans le cadre d'interventions dans la presse quotidienne nationale, d'une identité de "savant», concerne pour une part significative des individus eux-mêmes multipositionnés (Boltanski 1973) à l'intersection de différents champs, à commencer par le champ académique, le champ journalistique et le champ politique.

Par exemple, Dominique Reynié est agrégé et ancien membre du jury de l'agrégation de science politique, professeur à Sciences Po Paris, auteur ou directeur de nombreux ouvrages scientifiques ainsi que d'articles dans des revues centrales de la science politique en France (comme Raisons politiques et Politix); il est aussi un homme politique (classé à droite), directeur du think tank «La Fondation pour l'innovation politique » (Fondapol), expert auprès de la Commission européenne, ancien chroniqueur sur France culture ( Le monde selon Dominique Reynié ») et régulièrement invité dans des émissions télévisées comme $C$ dans l'air. 

les médias, on pourrait mentionner quelques individus ayant exercé comme journalistes (comme les politologues Jean-Paul Picaper et Alfred Grosser) et d'autres connus par ailleurs pour leur engagement partisan, comme le philosophe Yves Roucaute (qui a été membre de plusieurs cabinets ministériels de droite), le politologue Philippe Corcuff (ancien membre du Parti socialiste, des Verts, de la Ligue communiste révolutionnaire et actuellement membre de la Fédération anarchiste) ou le politologue Laurent Bouvet (représentant alors la «Gauche populaire ", un mouvement proche du PS, il a lancé depuis un mouvement « venu de la gauche $~^{17}$ focalisé sur la « défense de la laïcité » : le « Printemps républicain »).

En outre, dans certains cas, notamment pour une partie des spécialistes des questions européennes, le rapport au champ universitaire apparaît plus ténu ou indirect. C'est en particulier le cas des magistrats, parlementaires, fonctionnaires ou encore consultants qui, parce qu'ils interviennent également dans des institutions de l'enseignement supérieur - notamment dans des établissements prestigieux comme Sciences Po Paris -, peuvent faire valoir ce rattachement académique. Mais leurs différentes appartenances professionnelles sont parfois présentées de concert. Ainsi, la carrière politique de JeanLouis Bourlanges - député européen centriste - est évoquée en plus de son statut de «professeur associé à Sciences Po ». De même, Massimo Cacciari est présenté comme «philosophe» et «ex-maire de Venise »; et Yves Bertoncini, comme «directeur de Notre Europe-Institut Jacques Delors ${ }^{18}$ » et «enseignant au corps des Mines » [l'École des Mines est une prestigieuse école d'ingénieurs, accessible sur concours] ayant « consacré de nombreux ouvrages aux questions européennes ». À l'inverse, dans les cas des tribunes de Patrick Martin-Genier, Bruno Alomar, Nicolas Jean-Brehon ou encore Nicolas Bouzou, les signatures n'indiquent qu'une seule appartenance: «maîtres de conférence à l'IEP de Paris" pour les deux premiers (qui exercent respectivement comme magistrat et «conseiller en management»), "enseignant à [l'université] Paris 1 Panthéon-Sorbonne " pour le troisième (qui exerce de son côté surtout comme fonctionnaire parlementaire et chroniqueur au Monde de l'économie, qui est le supplément du journal Le Monde consacré aux questions économiques), «économiste» pour le dernier (qui est directeur-fondateur du cabinet de conseil Asteres et essayiste).

Ainsi, selon les cas, on manifeste le multi-positionnement, qui peut être source de prestige, notamment en cas de cumul de différents titres justifiant également l'accès à l'expression publique sur tel ou tel sujet; ou, à l'inverse, on le dissimule pour ne conserver que le capital symbolique associé au titre «savant ", notamment dans le cas d'auteurs moins connus, ou dont l'activité d'enseignement est mieux à même de légitimer l'expression publique que leurs autres titres.

\section{Conclusion}

La notion d'expertise présente donc plusieurs limites pour rendre raison des interventions dans la presse des chercheurs et universitaires en SHS en circonstance de campagne électorale. D'un côté, ces interventions, légitimées par une identité savante (voire par une spécialité thématique de recherche), évitent les déclarations d'affiliation partisane au profit de préconisations plus générales et donnent à voir des territoires d'expertise délimités en fonction des disciplines du savoir. Pour autant, si les 
journalistes semblent eux-mêmes encourager le modèle de "l'expert », en soulignant les titres savants à s'exprimer et en ouvrant les colonnes de leurs journaux à quelques auteurs relativement peu connus, d'autres contraintes et logiques entrent en jeu, favorisant les modèles plus classiques d'engagement. Par exemple, la logique de hiérarchisation des visibilités au sein de la presse généraliste favorise la surexposition d'un nombre réduit d'auteurs enclins eux-mêmes à intervenir sur des sujets variés et à se faire, si ce n'est "intellectuel universaliste " à la manière de Sartre, au moins « expert généraliste ».

De plus, en raison sans doute des contraintes proprement journalistiques, mais aussi des dispositions des auteurs enclins à s'y conformer, on remarque que les registres discursifs employés dans ces écrits publiés dans la presse généraliste ne se laissent que difficilement appréhender à partir de la catégorie d'expertise. Non seulement parce que la tribune d'opinion, genre qui fait appel à la liberté d'expression de l'intellectuel « universaliste ", reste, semble-t-il, le mode privilégié d'intervention des universitaires et chercheurs en SHS dans la presse quotidienne nationale. Mais aussi parce qu'on ne saurait, à l'examen de ce corpus d'articles, opposer radicalement la tribune d'opinion aux autres modalités d'intervention dans la presse écrite, comme l'entretien : chacun de ces formats favorise, de la part du lectorat, une adhésion doxique aux arguments proposés. En d'autres termes, afin de juger de la qualité de ces interventions, le lectorat du journal ne peut s'appuyer que sur la cohérence apparente de l'argumentation (c'està-dire sur ses propres catégories pré-constituées du jugement politique), sur sa disposition à l'égard des "savants" (considérés soit dans leur ensemble, soit individuellement en fonction de la pré-connaissance du nom propre de l'auteur), soit encore sur les connotations politiques associées à telle ou telle discipline. Dès lors, ces interventions dans la presse, "expertes» ou en tout cas légitimées comme telles, relèvent d'une captation du capital symbolique collectivement détenu par l'ensemble des universitaires et chercheurs en SHS, et rappellent corrélativement le risque de dévaluation de ce capital collectif que fait courir son usage individuel, lorsque le prophétisme est donné pour expertise.

\section{BIBLIOGRAPHIE}

ABBotT Andrew (1988). The System of Professions. An Essay on the Division of Expert Labor. Chicago, University of Chicago Press.

BOLTANSKI Luc (1973). «L'Espace positionnel : multiplicité des positions institutionnelles et habitus de classe ». Revue française de sociologie, 14(1) : 3-26.

BOURDIEU Pierre (1971). « Une Interprétation de la théorie de la religion selon Max Weber ». European Journal of Sociology/Archives Européennes de Sociologie/Europäisches Archiv für Soziologie, 12(1) : 3-21.

BOURDIEU Pierre (1984a). Homo academicus. Paris, Éditions de Minuit. 
BOURDIEU Pierre (1984b). « Le Hit-parade des intellectuels français ou qui sera juge de la légitimité des juges?». Actes de la recherche en sciences sociales, 52 : 95-100.

BOURDIEU Pierre (1988). L'Ontologie politique de Martin Heidegger. Paris, Éditions de Minuit. BOURDIEU Pierre (1996). Sur la télévision, suivi de L'emprise du journalisme. Paris, Liber Éditions. BOURDIEU Pierre (2002) [1989]. La Noblesse d'État : grandes écoles et esprit de corps. Paris, Éditions de Minuit, coll. « Le sens commun ».

BOURDIEU Pierre (2011). « Champ du pouvoir et division du travail de domination : texte manuscrit inédit ayant servi de support de cours au Collège de France, 1985-1986 ». Actes de la recherche en sciences sociales, $190: 126-139$.

BOUTIER Jean, PASSERON Jean-Claude, REVEL Jacques (2006). Qu'est-ce qu'une discipline. Paris, École des hautes études en sciences sociales, coll. « Enquête », 5.

CHARLE Christophe (1990). Naissance des « intellectuels », 1880-1900. Paris, Éditions de Minuit.

COLlovald Annie (1988). «Identité(s) stratégique(s) ». Actes de la recherche en sciences sociales, 73 : 29-40.

DELMAS Corinne (2006). Instituer des savoirs d'État. L'Académie des sciences morales et politiques. Paris, L'Harmattan.

DENORD François, LAGNEAU-YMONET Paul, THINE Sylvain (2011). « Le Champ du pouvoir en France ». Actes de la recherche en sciences sociales, 190 : 24-57.

DÉSINTOX (Association de citoyens contre la désinformation) (2004). Du Bon usage des tribunes du Monde. La Désinformation - La preuve, dossier 4, Paris.

DUCLERT Vincent (1999). «L'Engagement scientifique et l'intellectuel démocratique. Le sens de l'Affaire Dreyfus ». Politix, $48: 71-94$.

DULLER Matthias, KOROM Philipp, sCHÖGLER Rafael Y., FLECK Christian (2018). « Scholars as European public intellectuals ? Media interventions in the 2014 European Parliament election campaign ». European Societies, 20(2) : 322-353.

DUVAL Julien, GAUBERT Christophe, PAVIS Fabienne, LEBARON Frédéric, MARCHETTI Dominique (1998). Le « Décembre » des intellectuels français. Paris, Liber-Raisons d'Agir.

FRANÇOIS Bastien \& NEVEU Érik (1997). « Pour une sociologie politique des espaces publics contemporains ». In FRANçoIs Bastien \& NEVEU Erik (dir.). Espaces publics mosaïques. Acteurs, arènes et rhétoriques des débats publics contemporains. Rennes, Presses universitaires de Rennes : 13-58.

GARRIGOU Alain (2001). « La "Pensée Sciences Po" ». In Les Élites contre la République : Sciences Po et l'ENA. Paris, La Découverte : 79-112.

GAYON Vincent (2012). « Jeu critique : la "fin des intellectuels" (1975-1985)». Le Mouvement Social, $239: 25-44$

GINGRAS YVES (1991). «L'Institutionnalisation de la recherche en milieu universitaire et ses effets ». Sociologie et sociétés, 23(1): 41.

HEILBRON Johan (2004). « A regime of disciplines : toward a historical sociology of disciplinary knowledge ». In CAMIC Charles \& JOAS Hans (dir.). The dialogical turn: new roles for sociology in the postdisciplinary age. Lanham, MD, Rowman \& Littlefield : 23-42. 
JEANPIERRE Laurent (2016). « Tribunes et plateaux : logiques de la visibilité intellectuelle ». In CHARLE Christophe \& JEANPIERRE Laurent (dir.). La Vie intellectuelle en France, t. 2 (De 1914 à nos jours). Paris, Seuil : 494-500.

JEANPIERRE Laurent \& MOSBAH NATANSON Sébastien (2008). « French Sociologists and the Public Space of the Press: Thoughts Based on a Case Study (Le Monde, 1995-2002) ». In FLECK Christian, HESS Andreas, LYON E. Stina (dir.). Intellectuals and their Publics, Perspectives from the Social Sciences. Farnham/Burlington, Ashgate Publishing : 173-192.

JoLY Marc (2017). La Révolution sociologique. De la naissance d'un régime de pensée scientifique à la crise de la philosophie (XIX ${ }^{e}-\mathrm{XX}^{e}$ siècles). Paris, La Découverte.

LAFARGE Géraud \& MARCHETTI Dominique (2011). « Les Portes fermées du journalisme : L’espace social des étudiants des formations "reconnues" ». Actes de la recherche en sciences sociales, 189 : 72. LEBARON Frédéric (2017). « L'Économiste ou l'invention de “l'expert total” ». Bulletin du Snesup, $655: 14$.

LENSING-HEBBEN Caroline (2008). Les Experts cathodiques. Chercheurs face à la tentation médiatique. Paris, Le Bord de l'eau/INA.

MALHERBE Clément \& LUCE Raphaël (2017). « Invités des talk-shows et émissions de divertissement : tous les mêmes? La revue des médias ». La Revue des médias. (Consulté le 23 juillet 2020).

MATONTI Frédérique (2002). « La Colombe et les mouches. Frédéric Joliot-Curie et le pacifisme des savants ». Politix, $58: 109-140$.

MATONTI Frédérique \& SAPIRO Gisèle (dir.) (2009). « L'Engagement des intellectuels, nouvelles perspectives ». Actes de la recherche en sciences sociales, 176-177.

MAYAFFRE Damon (2005). « Les Corpus politiques : objet, méthode et contenu ». Corpus, 4. (Consulté le 23 juillet 2020).

MEMMI Dominique (1989). « Savants et maîtres à penser. La fabrication d'une morale de la procréation artificielle ». Actes de la recherche en sciences sociales, 76 : 82-103.

MERTON Robert K. (1968). « The Matthew Effect in Science ». Science, 3810 : 56-63.

NOIRIEL Gérard (2010). Dire la vérité au pouvoir : les intellectuels en question. Marseille, Agone.

PINTO Louis (2002). «L'Espace public comme construction journalistique. Les auteurs de "tribune" dans la presse écrite ». Agone, 26-27 : 151-182.

SAPIRO Gisèle (2009). « Modèles d'intervention politique des intellectuels : Le cas français ». Actes de la recherche en sciences sociales, 176-177 : 8-31.

SAPIRO Gisèle, BRUN Éric, FORDANT Clarisse (2018). « The Rise of the Social Sciences and the Humanities in France ; Institutionalization, Professionalization, Autonomization ». In FLECK Christian, DULLER Matthias, KARADY Viktor (dir.). Shaping Human Science Disciplines. Institutional Development in Europe and Beyond. Cham, Palgrave MacMillan : 25-68.

SIMÉANT Johanna (2002). « Friches, hybrides, et contrebandes : sur la circulation et la puissance militantes des discours savants ». In HAMMAN Philippe, MÉON Jean-Mathieu, VERRIER Benoît (dir.). Discours savants, discours militants : mélange des genres. Paris, L'Harmattan : 17-53.

Soulié Charles (2013). « Des Humanités à "l'économie de la connaissance” ? Les transformations du corps enseignant en lettres et sciences humaines en France (1949-2010) ». In CONESA Marc, 
LACOUR Pierre-Yves, ROUSSEAU Frédéric, THOMAS Jean-François (dir.). Faut-il brûler les Humanités et les Sciences humaines et sociales? Paris, Michel Houdiard Éditeur.

\section{NOTES}

1. Contrairement aux usages de la revue, notre article n'adopte pas la féminisation systématique pour souligner qu'il porte sur une population quasi exclusivement masculine (voir à ce sujet la section « S'exprimer dans la presse : une affaire d'hommes de pouvoir?»).

2. Dans cet article, nous définissons «l'expertise " non pas tant par le fait de répondre à une commande institutionnelle, mais plus largement comme un mode d'intervention publique s'effectuant au nom d'un savoir spécialisé et institué. En d'autres termes, ce que nous appelons ici « expert » se rapproche d'une figure avec laquelle on l'oppose parfois (Noiriel 2010), à savoir celle de « l'intellectuel spécifique » théorisée par Michel Foucault.

3. Par sciences humaines et sociales (SHS), nous entendons ici un ensemble de disciplines à prétention savante se différenciant des sciences dites « de la nature ». Cette catégorie d'origine institutionnelle présente certes l'inconvénient de placer sur un même plan des disciplines aux méthodes, objets et mêmes aux objectifs, parfois très différents. Si nous l'employons ici, c'est justement qu'il s'agira de comparer des universitaires ou chercheurs appartenant à différentes traditions disciplinaires ayant elles-mêmes en commun, a minima, une prétention savante et une même vocation à parler de la vie des humains en société (à travers des approches plus ou moins explicatives ou normatives, empiriques ou théoriques, etc.). Sont ainsi inclus dans notre corpus aussi bien des sociologues, politistes (ou «politologues» selon une appellation plus fréquente hors $\mathrm{du}$ monde académique), économistes, démographes, géographes, historiens, anthropologues, linguistes, mais aussi des philosophes ou encore des juristes.

4. Notion fréquemment invoquée dans le débat public, elle est pourtant absente du statut général des fonctionnaires en vigueur depuis 1983. Il s'agit en effet d'une construction de la jurisprudence administrative, reprise depuis dans certains statuts particuliers. Dans le cas des enseignants et chercheurs, leur indépendance et liberté d'expression est stipulée par l'article L. 952-2 du Code de l'éducation en vigueur à la date de rédaction du présent article : «Les enseignants-chercheurs, les enseignants et les chercheurs jouissent d'une pleine indépendance et d'une entière liberté d'expression dans l'exercice de leurs fonctions d'enseignement et de leurs activités de recherche, sous les réserves que leur imposent, conformément aux traditions universitaires et aux dispositions du présent code, les principes de tolérance et d'objectivité. »

5. La recherche menant à ces résultats a reçu des fonds de l'Union européenne issus de son septième programme-cadre (FP7/20072013) sous le numéro n 319974 (Interco-SSH).

6. Le Front national est le principal parti d'extrême-droite français. Depuis 2018, il se nomme Rassemblement national.

7. Le Parti socialiste est le principal parti de centre-gauche français.

8. Union pour la majorité présidentielle, puis Union pour un mouvement populaire, il s'agit du principal parti de centre-droit français. Il a pris le nom « Les Républicains » en 2015.

9. L'Observatoire du changement politique est une société privée chargée d'études de marché et de sondages, en particulier électoraux. Les dernières informations publiques sur l'entreprise datent de novembre 2016.

10. De même que pour la division des sujets abordés en fonction de la discipline, cette partition bien ordonnée souffre évidemment plusieurs exceptions ; par exemple la présence de NicolasJean Brehon - qui, formé en finances publiques, écrit une tribune d'analyse électorale - parmi les politologues.

11. Parmi les exceptions, on peut noter une tribune de Thomas Piketty parue dans Libération, : intitulée «Aux urnes, citoyen!", qui explicite une nette préférence de l'économiste pour le 
candidat Schulz. On relève aussi un appel à voter "pour l'Europe " émis dans un entretien accordé au Figaro par le politologue-journaliste Jean-Paul Picaper et l'avocat Alain Terrenoire.

12. La mention d'un ouvrage paru sur le sujet traité dans l'article est une manière d'importer cet appareil d'administration de la preuve. Encore faut-il noter que ces ouvrages se rangent le plus souvent, du point de vue de leurs auteurs eux-mêmes, dans la catégorie des essais plutôt que dans celle des écrits scientifiques.

13. Si parmi les soixante-dix-sept auteurs français ou exerçant en France de notre population, on exclut ceux qui ont obtenu une légion d'honneur, ceux qui sont présents dans le Who's who, et ceux qui sont intervenus au moins 50 fois entre 2000 et 2014 dans les médias audiovisuels français (au regard du nombre de renvois suscités par leur nom lorsqu'on interroge le catalogue de l'Institut national de l'audiovisuel [INA]), il ne nous reste plus que trente-et-un individus.

14. Project Syndicate se décrit comme la page de l'opinion du monde ("The World's Opinion Page »), et diffuse, en 13 langues, du contenu à caractère informatif produit par « des leaders politiques, des faiseurs de politiques publiques, des chercheurs, des leaders économiques et des activistes civiques du monde entier » (ibid.). Elle compte " 45 lauréats de prix Nobel et 111 chefs d'État et de gouvernement " parmi ses rédacteurs. Elle est - d'après son site internet principalement financée par des fonds de la fondation Bill and Melinda Gates, ou de la European Climate Foundation. On peut en première analyse la décrire comme un site de fabrication de la doxa internationale, avec un contenu tirant vers Courrier International pour un mode de production s'apparentant à celui de Vice.

15. Voir la présentation de la revue Le Débat sur le site des éditions Gallimard par Pierre Nora.

16. Par exemple, une grande partie des renvois pour Nicolas Bouzou concerne quelques émissions seulement, à savoir la «matinale : Teamtoussaint » et «La Newsroom » sur I-Télé, où il officie alors comme chroniqueur économique. Plus largement, il est fréquent qu'un grand nombre de renvois se concentre sur un petit groupe d'émissions.

17. Voir le « manifeste » du mouvement.

18. Il s'agit d'un « laboratoire d'idées» fondé par Jacques Delors en 1996 pour «alimenter le débat européen ».

\section{RÉSUMÉS}

L'analyse des interventions des chercheurs et universitaires en sciences humaines et sociales dans la presse quotidienne nationale française durant les élections européennes de mai 2014 permet d'interroger à nouveaux frais le développement de "l'expertise " comme forme d'engagement des intellectuels dans l'espace public. On pourrait s'attendre à ce que les savants privilégient une telle forme d'engagement, mais le font-ils effectivement lorsqu'ils interviennent dans la presse généraliste nationale? L'étude porte sur les articles qu'ils signent dans les journaux Le Monde, Libération et Le Figaro, qu'il s'agisse de tribunes, d'interviews ou encore de chroniques et d'articles d'analyse. En faisant une étude à la fois qualitative et quantitative du contenu de ces différentes productions, on révèle un ancrage des discours dans les disciplines d'appartenance des auteurs, mais également, et en particulier après les résultats de l'élection, une propension au prophétisme. Laissant peu d'espace à l'administration de la preuve, la légitimité de l'article de journal repose pourtant sur les compétences expertes arborées par son auteur et collectivement garanties par la catégorie professionnelle des chercheurs; dans ce cadre 
contraint, les usages individuels de ce capital collectif exposent du même coup au risque de son instrumentalisation et/ou de sa dévalorisation.

By analysing the interventions of humanities and social sciences (HSS) researchers and academics in national daily newspapers during the 2014 European electoral campaign, this article sheds new light on the development of "expertise" as a form of engagement of intellectuals in public space. While these scholars may be expected to lend their expertise, are they effectively doing this when they contribute to national generalist newspapers? This study focuses on the pieces they authored in the prominent newspapers Le Monde, Libération et Le Figaro - op-eds, interviews, columns, and analytical articles. The qualitative and quantitative study of the content of these productions reveals that discourses are rooted in the authors' disciplines, but also, especially after the election results, that they manifest an inclination towards prophecy. While they leave little room for well-argued demonstration, newspaper articles draw their legitimacy from the expert competencies displayed by the author and collectively guaranteed by researchers as a professional body. In this limited framework, this collective capital point is at risk of being instrumentalized and/or devalued when used individually.

\section{AUTEURS}

\section{CONSTANTIN BRISSAUD}

École des hautes études en sciences sociales (EHESS)/Centre européen de sociologie et de science politique-Centre européen de sociologie (Cessp-CSE)

\section{ÉRIC BRUN}

École des hautes études en sciences sociales (EHESS)/Centre européen de sociologie et de science politique-Centre européen de sociologie (Cessp-CSE) 